\title{
Neutrophil-Reduced Platelet Rich Plasma with Optimal Platelets Concentrations for Epicondylitis of the Elbow
}

\author{
M. Yoshida, M. Saito \\ Department of Orthopaedic Surgery, The Jikei University School of Medicine, Tokyo, Japan
}

\section{CORRESPONDING AUTHOR:}

Mamoru Yoshida,

Department of Orthopaedic Surgery

The Jikei University School of Medicine

3-25-8 Nishi-shinbashi

Minato-ku, Tokyo

105-8461 Japan

E-mail: mamoru@jikei.ac.jp

DOI:

10.32098/mltj.03.2021.10

LEVEL OF EVIDENCE: 5

\begin{abstract}
SUMMARY
Background. Autologous platelet-rich plasma (PRP) therapy has been applied in treatment of medial or lateral elbow epicondylitis; however, its efficacy is variable possibly due to differences in PRP platelet or neutrophil concentrations. We prepared neutrophil-reduced PRP with optimized platelet concentrations, and investigated therapeutic effects of the platelet-optimized PRP for elbow epicondylitis.

Methods. We treated 65 elbows of 60 patients ( 9 women and 51 men) with mean age of 50 years. Neutrophil-reduced PRP with optimized platelet concentrations were used in 41 lateral epicondylitis and 24 medial epicondylitis cases. Visual analogue pain scores (VAS) and modified Mayo Elbow Performance scores (MEPS) were obtained for each elbow at pre-PRP therapy and at 3, 6 or 12 months after the treatment.

Results. In 57 enthesopathies (34 lateral and 23 medial elbow epicondylitis), VAS or modified MEPS values at 3 months after treatment were significantly lower or higher, respectively, than those obtained before treatment; and this tendency was more manifested at 6 and 12 months after treatment. The PRP therapies were relatively less effective in 3 cases with complete tears at insertional site(s) of common tendons, 3 cases of lateral epicondylitis with synovial fringes, one with entrapment of the posterior interosseous nerve and in one welfare-reliant patient. Following the treatment with platelet-optimized PRP, VAS or modified MEPS values were lower or higher, respectively, than those reported in previous studies where not optimized PRP preparations were used. Conclusions. The platelet-optimized PRP therapy is effective for treatment of enthesopathy in elbow epicondylitis and this efficacy is higher than with PRP therapies without optimized platelet concentrations.
\end{abstract}

\section{KEY WORDS}

Platelet-rich plasma; lateral elbow epicondylitis; medial elbow epicondylitis; optimal concentration of platelets; enthesopathy.

\section{INTRODUCTION}

Medial or lateral elbow epicondylitis is a syndrome consisting of various pathophysiological disorders: enthesopathies of common flexor or extensor tendons, a complete full-thickness tears or partial tears of tendons, bursitis around elbow epicondyles, chronic damages of collateral ligaments or radial annular ligaments, synovitis of humeroradial joint, confirmed synovial folds in humeroradial joints or entrapment of the posterior interosseus nerve. The major pathophysiological change in elbow epicondyli- tis is enthesopathy characterized by tissue micro-damage or cellular apoptosis at the insertional site of tendons accompanied by abnormal angiogenesis and micro-shunts without inflammatory changes or reparative cellular reactions with resultant fibroblastic dysplasia (1). Autologous platelet-rich plasma (PRP) has been clinically used for treatment of various enthesopathies, i.e., medial or lateral elbow epicondylitis, Achilles tendinitis, plantar fasciitis or patellar tendinitis. Many studies demonstrated the therapeutic value of PRP in treatment of elbow epicondylitis, but its effects have been variable (2-12). Differences in platelet or leukocyte concen- 
tration or in proportions of leukocytes in PRPs prepared with different methods or commercial kits might have contributed to these different therapeutic outcomes. Since concentrations of platelets or leukocytes in PRP are dependent on their concentrations in the whole blood, variations in therapeutic effects are not unexpected and it has been reported that concentrations of platelets and/or leukocytes are different in preparations obtained in different institutions, and also in different patients using the same commercial preparation kits.

Platelets are the source of growth factors, cytokines and chemokines that regulate cellular anabolic processes and different platelet concentrations in PRP might influence the reparation ability of tendons necessary for the treatment of enthesopathy. The optimal concentration of platelets in $\mathrm{PRP}$ was found to range from $7.0 \times 10^{5} / \mu \mathrm{L}$ to $10.0 \times 10^{5} / \mu \mathrm{L}$ in the animal model (13) and this finding was supported by several in vitro or in vivo studies (14-16).

It is well known that leukocyte concentrations also influence the therapeutic efficacy of PRP, and that neutrophils that comprise approximately $50 \%$ of all leukocytes in the peripheral blood, are mainly involved in the process of inflammation, especially tissue degradation. Dragoo demonstrated that PRP rich in leukocytes initiated a strong inflammatory cellular reaction in rabbit tendons at 5 days after PRP injection (17). McCarrell showed that PRP with a high concentration of leukocytes induced higher expressions of pro-inflammatory cytokines and catabolic mediators as well as suppressed collagen synthesis (18). During treatment of enthesopathy, neutrophils inhibited the restoration process since the extracellular matrix was damaged during inflammation and lower synthesis of collagen suppressed the formation of tendon matrix. These negative influences of neutrophils in PRP therapy were also demonstrated in several studies and to obtain excellent post-PRP therapy recovery, exclusion of neutrophils from PRP has been recommended (19-24).

We optimized the PRP for the treatment of enthesopathy and maintained platelet concentrations within the range from $7.0 \times 10^{5} / \mu \mathrm{L}$ to $10.0 \times 10^{5} / \mu \mathrm{L}$ and with approximately $90 \%$ of neutrophils excluded. We used these optimized PRPs in treatment of medial or lateral elbow epicondylitis and present the obtained results.

\section{PATIENTS AND METHODS}

The present study was conducted according to the journal's guidelines (25). Informed consent was obtained from each patient for the use of personal medical records and approval for the study was obtained from the institutional ethics committee (\#2020-9).
Therapies with platelet-optimized PRPs were applied in 65 elbows [ 60 patients, 51 men and 9 women; mean age of 50 years (range, 15-80 years)], including 41 lateral epicondylitis and 24 medial epicondylitis cases. The mean period from the diagnosis to the commencement of PRP therapy was 22.8 months (range, 3-120 months), and the mean duration of follow-up was 14 months (range, 3-36 months). Lateral or medial elbow epicondylitis were diagnosed based on chief complains and/or physical examinations of each patient according to the following inclusion criteria: 1) pain occurred at the lateral or medial elbow epicondyle during active motion of wrists or fingers, or in the resting state; 2) tenderness at the lateral or medial epicondyle of the elbows was present; and 3) a lateral or medial elbow pain was induced upon resistance against active dorsi-flexion (Thomsen test) or volar flexion of the wrist. There were 44 cases of elbow epicondylitis related to sports injuries (21 golf, 7 baseball, 5 tennis, 4 arm wrestling, 1 boxing, 1 table tennis, 1 surfing, 1 bouldering, 1 kendo, 1 kyudo, 1 gym work), 7 epicondylitis cases were caused by manual labor, 6 epicondylitis cases were related to keyboard typing, 4 were due to house-keeping jobs, 3 were caused by agriculture work, and in one case epicondylitis developed after reparative surgeries for full-thickness complete tears of the medial proximal flexor common tendon performed at a different institution. We examined plain X-ray images and magnetic resonance images (MRI) in all elbows and found high intensity areas at the insertional sites of tendons in T2-weighted MRI or short tau inversion recovery (STIR) modes in 21 elbows, obvious synovial fringes of humeroradial joints in 3 elbows, complete full-thickness tears of common extensor tendons at the lateral epicondyles in 2 elbows, or a complete tear of the common flexor tendon at the medial epicondyle in one elbow of the patient after a previous surgery. We excluded cases affected with the arthritis or the osteoarthritis of elbows, rheumatoid arthritis, post-injury deformations of elbows, injuries or damages of extensor muscles located at forearm or cervical spondylo-radiculopathies, of which symptoms medial or lateral elbow pain was involved in for the correction of cases affected with enthesopathies or terminal stage of enthesopathies (26). A visual analog pain scale (VAS) or a modified Mayo Elbow Performance score (MEPS) were obtained for each elbow before the PRP therapies and at 3, 6 or 12 months after completion of the treatment. The student $t$ test was used to statistically compare the before and after scores. Before starting the PRP therapy, the average VAS score was $68.1 \pm 5.5 \mathrm{~mm}$ and the average modified MEPS score was $57.9 \pm 14.2$ points.

Approximately $3.0 \mathrm{~mL}$ of the autologous leukocyte-reduced PRP was obtained from $22 \mathrm{~mL}$ of the whole blood with a preparation kit (MyCells, Kaylight, Tel Aviv, Israel) accord- 
ingly with the manufacturer's instructions. Briefly, the whole blood was centrifuged for 7 minutes at $2000 \times \mathrm{g}$. After aspiration of the supernatant plasma, the approximate $3.0 \mathrm{~mL}$ of the residual plasma was agitated to disperse the platelets, which were then precipitated on the separation gel. This plasma was filtered to exclude the debris and approximately 3.0 $\mathrm{mL}$ of filtered non-activated PRP was obtained. Concentrations of platelets or neutrophils in the prepared PRPs were measured with an automated blood cell counter and analyzer in each 10 -fold diluted sample prepared by mixing $100 \mu \mathrm{L}$ of the PRP with $900 \mu \mathrm{L}$ of the normal saline. Platelet concentrations were confirmed to be in the range between $7.0 \times 10^{5} /$ $\mu \mathrm{L}$ and $10.0 \times 10^{5} / \mu \mathrm{L}$. Approximately $90 \%$ of neutrophils were removed by the separation gel present in the primary spit of the MyCells kit. The mechanism for exclusion of neutrophils was not revealed by the kit's manufacturer. The PRP prepared in the present study was classified as type 3 in Mishra's classification and as pure PRP in the PAW classification since the PRP was not activated before injection $(27,28)$. The prepared PRPs were slowly and gently injected into the points of the most severe tenderness at the lateral or medial elbow epicondyles through a $30 \mathrm{G}$ needle under ultrasonographic guidance without previous anesthesia and after sterilization of the skin surface with a $10 \%$ povidone-iodine solution. Activities of daily living were permitted after injections and at 3 weeks, and efficacy of the PRP therapy was evaluated through clinical examination. When the therapeutic effect was not considered satisfactory, i.e., the sufficient reduction in pain was not observed, the second PRP injection was carried out in the same manner. Three weeks later, another clinical examination was performed and the treatment efficacy was evaluated. If during the observation course, the pain initially improved but could not be completely eliminated, another (the $3^{\text {rd }}$ ) injection of the PRP was performed. In all patients, clinical follow-up had been carried out for 3-36 months following the initial injections. Moreover, physical conditioning programs were implemented during the PRP therapy for patients willing to return to sports activities when they also had other physical problems that potentially interfered with their sports activity, such as limited motion range in the shoulder, hip and/or spinal joints.

\section{RESULTS}

Platelet concentrations in all prepared PRPs ranged between $7.0 \times 10^{5}$ to $9.6 \times 10^{5} / \mu \mathrm{L}$ and their average number was $8.2 \pm$ $0.7 \times 10^{5} / \mu \mathrm{L}$. The average leukocyte concentration was 3100 $\pm 360 / \mu \mathrm{L}$ and the average neutrophil concentration was 250 $\pm 30 / \mu \mathrm{L}$. Almost all erythrocytes and approximately $90 \%$ of neutrophils were removed from the PRPs. Hence, the prepared PRPs were classified as type 3 according to the Mish- ra's classification and as the pure PRP according to the PAW classification, since they were not activated before injections.

The overall average number of PRP injections was 1.9 (range, 1-9) times. At 3 months after PRP injections, the average value of VAS in all elbows was $26.7 \pm 15.2 \mathrm{~mm}$, which was significantly lower than that before injections $(\mathrm{p}<$ 0.01 ) and the average modified MEPS value in all elbows was $86.7 \pm 20.6$ points, significantly higher than that before injections $(\mathrm{p}<0.01)$. However, poor recoveries were observed in 2 elbows with complete full-thickness tears of the common extensor tendons, in one elbow with a complete tear of the common flexor tendon following a previous surgery, in 3 elbows with lateral epicondylitis accompanied by synovial fringes in humeroradial joints, in one elbow with lateral epicondylitis accompanied by an entrapment disorder of the posterior interosseus nerve, and in one welfare-reliant patient with lateral epicondylitis. In these cases, poor PRP treatment effects did not improve even after 2 to 4 PRP injections. The VAS or modified MEPS scores in these 8 elbows were significantly higher or lower, respectively, than those of the other 57 elbows at 3 months after the PRP therapies. Therefore, in these 8 cases, the PRP therapies were terminated. In 2 complete full-thickness tears of the common extensor tendons reparation surgeries using surgical anchors were performed. In 3 lateral epicondylitis cases with synovial fringes, arthroscopic resections of the fringes were performed, and in one lateral epicondylitis case with entrapment of the posterior interosseus nerve, neurolysis surgery was carried out in a different institution. Patients followed at our institution after surgery ( 6 elbows) returned to their previous activities at 3 to 6 months after operation. The final outcomes of 2 other patients with poor recovery after PPR therapy were unknown because information was not available: one patient with the complete tear of the common flexor tendon after surgery performed at a different institution was transferred back there and underwent a re-reparative surgery, and another, the welfare-reliant patient, was transferred to a different hospital and underwent a shockwave therapy.

In the remaining 57 elbows, the average VAS value was 21.4 $\pm 5.1 \mathrm{~mm}$ (improved by $68.5 \%$ ) and the average modified MEPS value was $90.9 \pm 3.6$ points at 3 months after PRP injections. All 52 patients with 57 elbows returned to their pre-injury activities at the mean of 12 (range, 4-20) weeks after initial PRP injections. At 6 months after injections, the average VAS value in 57 elbows was $9.4 \pm 6.2 \mathrm{~mm}(86.2 \%$ improvement) and the average modified MEPS value was $95.0 \pm 3.9$ points. At 12 months after injections, the average VAS value in 55 elbows (the follow up was shorter than 12 months in 2 cases) was $4.9 \pm 4.2 \mathrm{~mm}$ and the average modified MEPS value in these 55 elbows was $97.4 \pm 3.8$ points (figures $\mathbf{1}, \mathbf{2}$ ). These VAS and modified MEPS values 
were significantly lower or higher, respectively, than those before the PRP therapy at 3, 6 or 12 months after the therapy ( $\mathrm{p}<0.01)$. At 12 months, VAS or modified MEPS values were significantly lower or higher, respectively, than those at 3 months after the PRP therapy. Moreover, there were no statistical differences in VAS or MEPS values between 34 elbows of lateral epicondylitis and 23 elbows of medial epicondylitis at 3 or 6 months after injections, and between

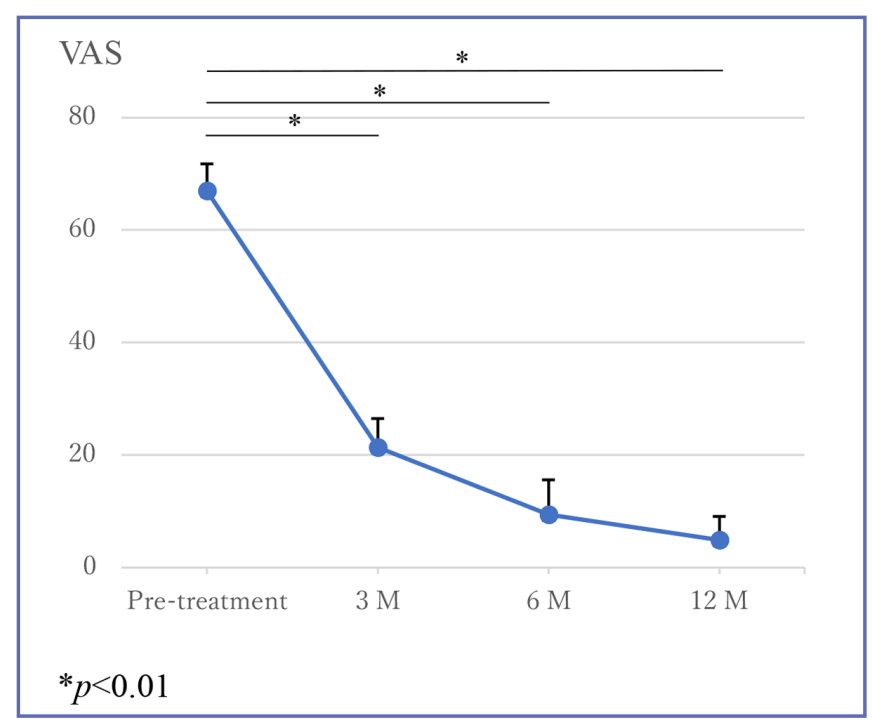

Figure 1. Visual Analogue Scale (VAS) values of 57 elbows (34 lateral and 23 medial epicondylitis) at pre-PRP therapy and at 3,6 or 12 ( $n=55,33$ lateral and 22 medial) months after the PRP therapy were plotted in the graph. *VAS values at 3,6 or 12 months after the therapy were significantly lower than those at pre-therapy $(p<0.01)$.
33 lateral and 22 medial epicondylitis at 12 months after injections. Neither adverse events nor recurrences were observed at any time point during the follow-up 18 months in the present study. Numbers or area sizes of high intensity areas observed in MR images disappeared or declined in 9 elbows after the PRP therapy at 6 to 12 months (figure 3), and no obvious MRI changes were observed after the PRP therapy in 6 elbows among 15 elbows examined.

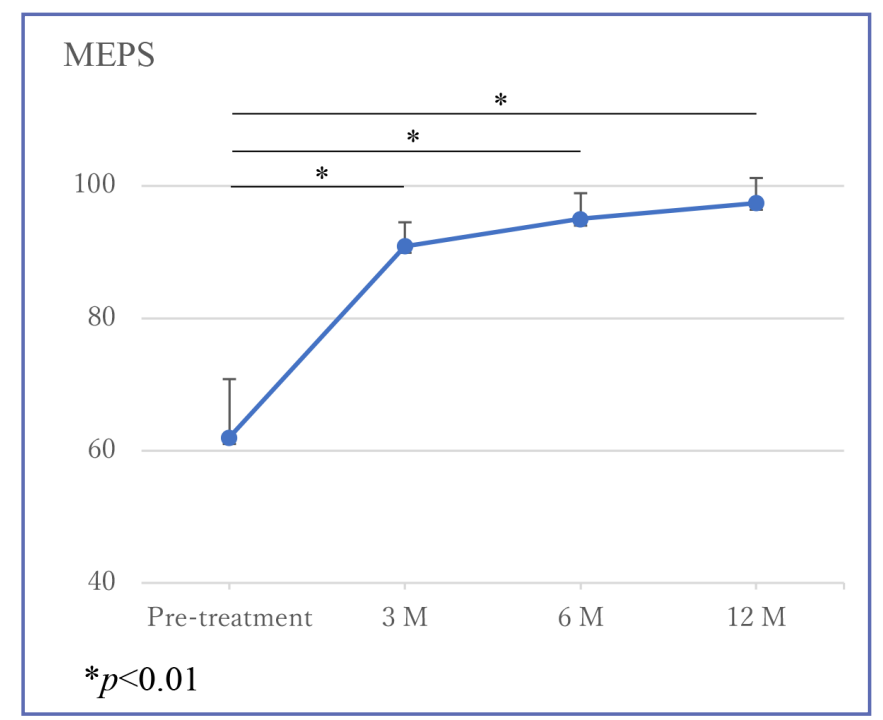

Figure 2. Modified Mayo Elbow Performance Score (MEPS) values of 57 elbows (34 lateral and 23 medial epicondylitis) at pre-PRP therapy and at 3, 6 or $12(n=55,33$ lateral and 22 medial) months after the PRP therapy were plotted in the graph. *Modified MEPS values at 3,6 or 12 months after the therapy were significantly higher than those at pre-therapy $(p<0.01)$.
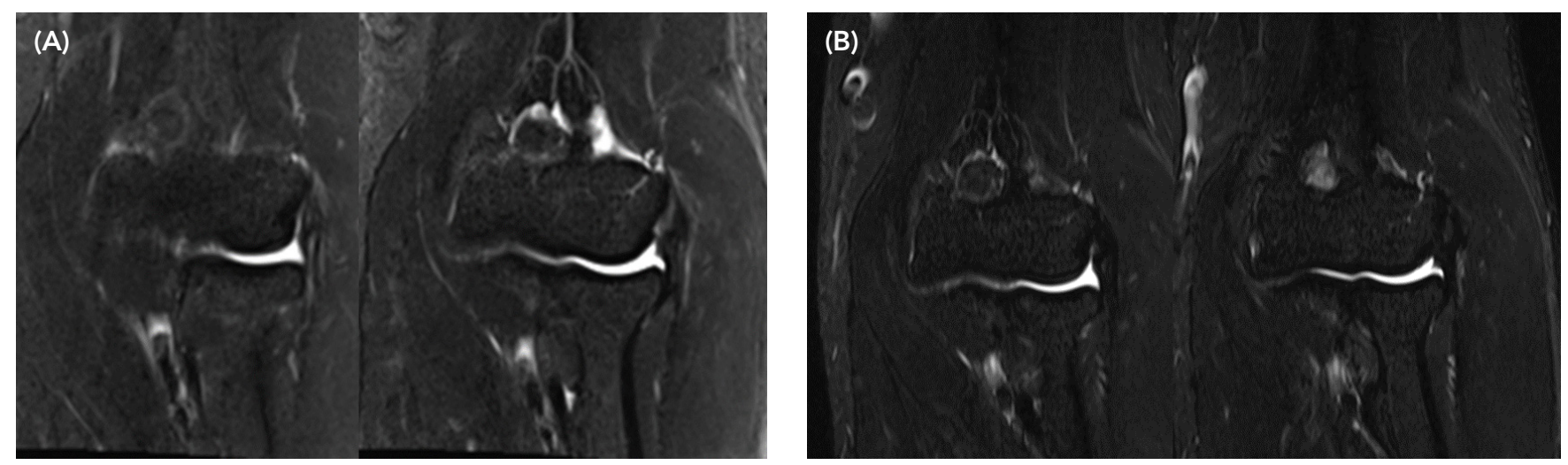

Figure 3. (A) Magnetic resonance coronal sections of the left elbow of a 53-year-old male with lateral epicondylitis in the short tau inversion recovery (STIR) mode. High intensity areas were observed at the insertional site of the common extensor tendon at pre-PRP therapy. (B) High intensity areas almost disappeared after the PRP therapy. 


\section{DISCUSSION}

Several studies investigated therapeutic effects of PRP in the treatment of enthesopathies, including elbow epicondylitis, patellar tendinopathies or plantar fasciitis; however, in most of those studies, concentrations of platelets or neutrophils in the injected PRPs were not reported and it was possible that these concentrations were not optimal. In the present case series, we demonstrated that platelet or neutrophil concentrations in all prepared PRPs could be measured, that platelet-optimized PRP for treatment of enthesopathy could be prepared with commercially available kits, and that the treatment with the platelet-optimized PRP was safe and effective for enthesopathies of elbow epicondylitis. Similar studies on the therapeutic effects of platelet-optimized PRPs have not been performed; therefore, our series is the first demonstrating satisfactory results of platelet-optimized PRP therapy in the treatment of elbow epicondylitis. Raeissadat SA et al. (29) conducted a prospective double-blinded randomized control study (RCT), in which 31 lateral epicondylitis cases were treated with PRPs with mean platelet concentration of 12.27 $\pm 2.50 \times 10^{5} / \mu \mathrm{L}$ and mean white blood cells concentration of $6740 \pm 1396 / \mu \mathrm{L}$ (neutrophil concentrations unknown). They showed that post-treatment VAS values were $29.1 \pm 24.7$ $\mathrm{mm}$ at 6 months and $32.9 \pm 24.1 \mathrm{~mm}$ at 12 months and that modified MEPS values were $81.2 \pm 16$ at 6 months and 78.18 \pm 18 at 12 months after the PRP therapy. In a prospective RCT, Mishra AK et al. (19) also treated 101 lateral epicondylitis with leukocyte-enriched PRP (the mean concentration of leukocytes or neutrophils were unknown) where $5 \times$ baseline concentrations of non-activated platelets were used, and showed the mean improvement of $55.1 \%$ in VAS scores at 3 months and of $71.5 \%$ at 6 months after the PRP therapy; however, these values were not significantly different with those of controls. In these two studies, concentrations of platelets or neutrophils in the injected PRPs were out of the optimal ranges. In our study, the mean VAS or modified MEPS values at 3, 6 or 12 months after PRP therapies were respectively lower or higher than in these two studies with not-optimized platelet concentrations, when all respective mean values were compared. This finding indicated that efficacy of the PRP therapy with optimized platelet concentrations in treatment of elbow epicondylitis was higher than that of the PRP therapy without optimized platelet concen-

\section{REFERENCES}

1. Vaquero-Picado A, Barco R, Antuña SA. Lateral epicondylitis of the elbow. EFORT Open Rev 2017;1(11):391-7.

2. Mishra A, Pavelko T. Treatment of chronic elbow tendinosis with buffered platelet-rich plasma. Am J Sports Med 2006;34(11):1774-8. trations, and that control of platelet and neutrophil concentrations in PRP preparations is important or even essential to obtain an excellent recovery.

The present case series demonstrated that the PRP therapy was not effective in cases with complete full-thickness tears of the common flexor or extensor tendons or in cases of lateral elbow epicondylitis accompanied with synovial fringes in the humeroradial joints, notwithstanding the optimized platelet or neutrophil concentrations in injected PRPs, and that reparative surgeries or arthroscopic resections of synovial fringes, respectively, were successful in those cases.

Limitations of the present study are: the lack of control group, small sample number, short experimental period and low rates of MRI examinations after PRP therapies. Further studies are necessary to prove the necessity for optimizing platelet or leukocyte concentrations in PRPs used in the treatment of elbow epicondylitis.

\section{CONCLUSIONS}

A therapy with platelet-optimized PRP, in which concentrations of platelets and neutrophils are maintained in optimal ranges, was proven to be a safe method available for the treatment of elbow epicondylitis enthesopathies. A tight control of platelet and neutrophil concentrations seems to be important or even essential if high therapeutic efficacy of the PRP therapy is to be obtained. The complete tears of the insertional site of the common tendon being resistant to the PRP therapy require reparative surgeries and arthroscopic resection is often necessary for treatment of lateral elbow epicondylitis accompanied with synovial fringes in the humeroradial joints.

\section{ACKNOWLEDGEMENTS}

We would like to express our grateful thanks to all staff working in the blood laboratory of the hospital for their kind corporation to measure the concentrations of platelets, leukocytes or neutrophils in PRPs.

\section{CONFLICT OF INTERESTS}

The authors declare that they have no conflict of interests. 
4. Gosens T, Peerbooms JC, van Laar W, den Oudsten BL. Ongoing positive effect of platelet-rich plasma versus corticosteroid injection in lateral epicondylitis: A double-blind randomized controlled trial with 2-year follow-up. Am J Sports Med 2011;39(6):1200-8.

5. Thanasas C, Papadimitriou G, Charalambidis C, Paraskevopoulos I, Papanikolaou A. Platelet-rich plasma versus autologous whole blood for the treatment of chronic lateral elbow epicondylitis: a randomized controlled clinical trial. Am J Sports Med 2011;39(10):2130-4.

6. Kwapisz A, Prabhakar S, Compagnoni R, Sibilska A, Randelli P. Platelet-rich plasma for elbow pathologies: a descriptive review of current literature. Curr Rev Musculoskelet Med 2018;11(4):598-606.

7. Chen X, Jones IA, Park C, Vangsness CT Jr. The efficacy of platelet-rich plasma on tendon and ligament healing: a systematic review and meta-analysis with bias assessment. Am J Sports Med 2018;46(8):2020-32.

8. Houck DA, Kraeutler MJ, Thornton LB, McCarty EC, Bravman JT. Treatment of lateral epicondylitis with autologous blood, platelet-rich plasma, or corticosteroid injections: a systematic review of overlapping meta-analyses. Orthop J Sports Med 2019;14;7(3):2325967119831052.

9. Huang K, Giddins G, Wu LiD. Platelet-rich plasma versus corticosteroid injections in the management of elbow epicondylitis and plantar fasciitis: an updated systematic review and meta-analysis. Am J Sports Med 2019;10:363546519888450.

10. Xu Q, Chen J, Cheng L. Comparison of platelet rich plasma and corticosteroids in the management of lateral epicondylitis: A meta-analysis of randomized controlled trials. Int J Surg 2019;67:37-46.

11. Dragoo JL, Meadows MC. The use of biologics for the elbow: a critical analysis review. J Shoulder Elbow Surg. 2019;28(11):2053-60.

12. Li A, Wang H, Yu Z, et al. Platelet-rich plasma vs corticosteroids for elbow epicondylitis: a systematic review and meta-analysis. Medicine (Baltimore) 2019;98(51):e18358.

13. Yoshida M, Funasaki H. Optimal platelet concentration for the therapeutic effect of autologous neutrophil-reduced platelet rich plasma in a rat model of Achilles tendinopathy. Muscles Ligaments Tendons J 2020;10(4):612-21.

14. Boswell SG, Schnabel LV, Mohammed HO, et al. Increasing platelet concentrations in leukocyte-reduced platelet-rich plasma decrease collagen gene synthesis in tendons. Am J Sports Med 2014;42(1):42-9.

15. Giusti I, D’Ascenzo S, Manco A, et al. Platelet concentration in platelet-rich plasma affects tenocyte behavior in vitro. BioMed Res Int 2014;2014:630870.

16. Yoshida M, Funasaki H, Marumo K. Efficacy of autologous leukocyte-reduced platelet-rich plasma therapy for patellar tendinopathy in a rat treadmill model. Muscles Ligaments Tendons J 2016;6(2):205-15.
17. Dragoo JL, Braun HJ, Durham JL, et al. Comparison of the acute inflammatory response of two commercial platelet-rich plasma systems in healthy rabbit tendons. Am J Sports Med 2012;40:1274-81.

18. McCarrel TM, Minas T, Fortier LA. Optimization of leukocyte concentration in platelet-rich plasma for the treatment of tendinopathy. J Bone Joint Surg Am 2012;94(19):e143(1-8).

19. Mishra AK, Skrepnik NV, Edwards SG, et al. Efficacy of platelet-rich plasma for chronic tennis elbow: a doubleblind, prospective, multicenter, randomized controlled trial of 230 patients. Am J Sports Med 2014;42:463-71.

20. Zhou Y, Zhang J, Wu H, Hogan MV, Wang JH-C. The differential effects of leukocyte-containing and pure platelet-rich plasma (PRP) on tendon stem/progenitor cells implications of PRP application for the clinical treatment of tendon injuries. Stem Cell Res Ther 2015;15(6):173.

21. Cross JA, Cole BJ, Spatny KP, et al. Leukocyte-reduced platelet-rich plasma normalizes matrix metabolism in torn human rotator cuff tendons. Am J Sports Med 2015;43(12):2898-906.

22. Yan R, Gu Y, Ran J, et al. Intratendon delivery of leukocyte-poor platelet-rich plasma improves healing compared with leukocyte-rich platelet-rich plasma in a rabbit Achilles tendinopathy model. Am J Sports Med 2017;45(8):1909-20.

23. Yerlikaya M, Çaliş HT, Sütbeyaz ST, et al. Comparison of effects of leukocyte-rich and leukocyte-poor platelet-rich plasma on pain and functionality in patients with lateral epicondylitis. Arch Rheumatol 2017;16;33(1):73-9.

24. Noh KC, Liu XN, Zhuan Z, et al. Leukocyte-poor platelet-rich plasma-derived growth factors enhance human fibroblast proliferation in vitro. Clin Orthop Surg 2018;10(2):240-7.

25. Padulo J, Oliva F, Frizziero A, Maffulli N. Muscles, Ligaments and Tendons Journal - Basic principles and recommendations in clinical and field Science Research: 2018 update. Muscles Ligaments Tendons J 2018;8(3):305-7.

26. Di Filippo L, Pennella D, Maselli F, Arrigoni P. Research proposal of a new clinic model for the interpretation of Lateral Elbow Pain: is it time to change? Muscle Ligaments Tendons J 2020;10(1):57-66.

27. Dohan Ehrenfest DM, Andia I, Zumstein MA, et al. Classification of platelet concentrates (Platelet-Rich Plasma-PRP, Platelet-Rich Fibrin-PRF) for topical and infiltrative use in orthopedic and sports medicine: current consensus, clinical implications and perspectives. Muscles Ligaments Tendons J 2014;4(1):3-9.

28. Kaux J-F, Bouvard M, Lecut C, et al. Reflections about the optimisation of the treatment of tendinopathies with PRP. Muscle Ligaments Tendons J 2015;5(1):1-4

29. Raeissadat SA, Rayegani SM, Hassanabadi H, et al. Is Platelet-rich plasma superior to whole blood in the management of chronic tennis elbow: one year randomized clinical trial. BMC Sports Sci Med Rehabil 2014;6:12. 